\title{
CONVERGENCE OF SERIES OF GAUSSIAN MARKOV SEQUENCES
}

UDC 519.21

\author{
M. K. RUNOVSKA
}

\begin{abstract}
We find necessary and sufficient conditions for the almost sure convergence of sums of centered Gaussian Markov sequences.
\end{abstract}

\section{INTRODUCTION}

Consider a sequence of random variables $\left(\xi_{k}\right)=\left(\xi_{k}, k \geq 1\right)$ defined by the system of recurrence equations

$$
\xi_{0}=0, \quad \xi_{k}=\alpha_{k} \xi_{k-1}+\beta_{k} \theta_{k}, \quad k \geq 1
$$

where $\left(\alpha_{k}\right)$ is a sequence of real numbers, $\left(\beta_{k}\right)$ a sequence of nonnegative numbers, and $\left(\theta_{k}\right)$ a sequence of independent symmetric random variables such that $\mathrm{P}\left\{\theta_{k}=0\right\}<1$, $k \geq 1$. Necessary and sufficient conditions for the almost sure (a.s.) convergence of the random series $\sum_{k=1}^{\infty} \xi_{k}$ are obtained in the paper [4.

In this paper, we consider a class of centered Gaussian Markov sequences $\left(\xi_{k}\right)$ or, in other words, sequences of random variables defined by the system of recurrence relations

$$
\xi_{0}=0, \quad \xi_{k}=\alpha_{k} \xi_{k-1}+\beta_{k} \gamma_{k}, \quad k \geq 1,
$$

where $\left(\alpha_{k}\right)$ is a sequence of real numbers, $\left(\beta_{k}\right)$ a sequence of nonnegative numbers, and $\left(\gamma_{k}\right)$ a standard Gaussian sequence, that is, a sequence of independent Gaussian random variables with zero mean and unit variance.

The aim of this paper is to find conditions for the almost sure convergence of the random series

$$
\sum_{k=1}^{\infty} \xi_{k}
$$

for a centered Gaussian Markov sequence $\left(\xi_{k}, k \geq 1\right)$ defined by (11). Note that the asymptotic properties of realizations of Gaussian Markov sequences are studied in the paper [1]. In particular, necessary and sufficient conditions for the almost sure convergence to zero are obtained in [1] for the case of Gaussian Markov sequences.

An interested reader is invited to consult the paper [4, since we use the main notation introduced in [4].

\section{Auxiliary Results}

Consider a finite-dimensional space $\mathbb{R}^{d}, d \geq 1$. Let $c\left(\mathbb{R}^{d}\right)$ be the space of all convergent sequences belonging to the space $\mathbb{R}^{d} ;\left(X_{n}\right)$ a sequence of independent symmetric random

2010 Mathematics Subject Classification. Primary 60G50, 65B10, 60G15; Secondary 40 A05.

Key words and phrases. Gaussian Markov sequence of random variables, almost sure convergence of random series, theory of sums of independent random elements with operator normalizations. 
vectors of the space $\mathbb{R}^{d} ; \Xi_{n}=\sum_{k=1}^{n} X_{k}, n \geq 1$; and let $\left(\mathcal{A}_{n}\right)$ be a sequence of linear continuous operators acting from $\mathbb{R}^{d}$ to $\mathbb{R}^{d}$. The class of all monotone sequences of positive integer numbers tending to infinity is denoted by $\mathfrak{R}^{\infty}$.

Below we give a criterion for the almost sure convergence of sums of independent symmetric random vectors with an operator normalization in the space $\mathbb{R}^{d}$ (see [2, 3]).

Theorem 2.1. The relation

$$
\left(\mathcal{A}_{n} \Xi_{n}\right) \in c\left(\mathbb{R}^{d}\right) \quad \text { a.s. }
$$

is equivalent to the set of the following three conditions:

A) for all $k \geq 1$,

$$
\left(\mathcal{A}_{n} X_{k}\right) \in c\left(\mathbb{R}^{d}\right) \quad \text { a.s.; }
$$

B) the series $\sum_{k=1}^{\infty} Y_{k}$ converges in $\mathbb{R}^{d}$ almost surely, where

$$
Y_{k}=\lim _{n \rightarrow \infty}\left(\mathcal{A}_{n} X_{k}\right) ;
$$

C) for all sequences $\left(m_{j}\right)$ of the class $\mathfrak{R}^{\infty}$,

$$
\left\|\mathcal{A}_{m_{j+1}}\left(\Xi_{m_{j+1}}-\Xi_{m_{j}}\right)\right\| \underset{j \rightarrow \infty}{\longrightarrow} 0 \quad \text { a.s. }
$$

We use the following result [2, 3] to check condition C) of Theorem 2.1 in the case of Gaussian random vectors.

Lemma 2.1. Let $\left(\Gamma_{k}\right)$ be a sequence of centered Gaussian random vectors in the space $\mathbb{R}^{d}$, $d \geq 1$. If

$$
\sum_{k=1}^{\infty} \exp \left\{-\frac{\varepsilon}{\mathrm{E}\left\|\Gamma_{k}\right\|^{2}}\right\}<\infty
$$

for all $\varepsilon>0$, then

$$
\lim _{k \rightarrow \infty}\left\|\Gamma_{k}\right\|=0 \quad \text { a.s. }
$$

If $\left(\Gamma_{k}\right)$ is a sequence of independent random vectors, then relations (3) and (4) are equivalent.

\section{A CRITERION FOR THE CONVERGENCE OF SUMS \\ of Gaussian Markov SEQUences}

Let $n \geq 1$ and

$$
A^{\prime}(n, k)= \begin{cases}1+\sum_{l=1}^{n-k}\left(\prod_{j=k+1}^{k+l} \alpha_{j}\right), & 1 \leq k \leq n-1, \\ 1, & k=n, \\ 0, & k>n,\end{cases}
$$

and

$$
A(n, k)=\beta_{k} A^{\prime}(n, k), \quad n, k \geq 1 .
$$

For $k \geq 1$, put

$$
A(\infty, k)=\beta_{k}+\sum_{l=1}^{\infty}\left(\beta_{k} \prod_{j=k+1}^{k+l} \alpha_{j}\right) .
$$


Note that $A(\infty, k)=\lim _{n \rightarrow \infty} A(n, k)$ if the limit exists. The latter condition means that the series

$$
\sum_{l=1}^{\infty} \beta_{k}\left(\prod_{j=k+1}^{k+l} \alpha_{j}\right)
$$

converges. Below is the criterion for the almost sure convergence of series (2) with the terms defined by (1).

Theorem 3.1. The random series (2) converges almost surely if and only if the following three conditions hold:

1) series (8) converges for all $k \geq 1$;

2) the following relation holds:

$$
\sum_{k=1}^{\infty}(A(\infty, k))^{2}<\infty
$$

3) for all $\varepsilon>0$ and all sequences $\left(m_{j}\right)$ of the class $\mathfrak{R}^{\infty}$,

$$
\sum_{j=1}^{\infty} \exp \left\{-\frac{\varepsilon}{\sum_{i=m_{j}+1}^{m_{j+1}}\left(A\left(m_{j+1}, i\right)\right)^{2}}\right\}<\infty .
$$

Proof. First we introduce some notation. Consider a sequence $\left(S_{n}\right)$ of partial sums of series (2):

$$
S_{n}=\sum_{k=1}^{n} \xi_{k}, \quad n \geq 1 .
$$

The method of the proof of Theorem 3.1 is to pass from the sequence $\left(S_{n}\right)$ to the sequence of sums of independent centered Gaussian vectors in the space $\mathbb{R}^{2}$. This method was introduced in 2, 3].

Since $\xi_{n}=S_{n}-S_{n-1}, n \geq 1$, relation (11) implies that the sequence $\left(S_{n}\right)$ satisfies the recurrence relation of the second order, namely

$$
S_{-1}=S_{0}=0, \quad S_{n}=\left(1+\alpha_{n}\right) S_{n-1}-\alpha_{n} S_{n-2}+\beta_{n} \gamma_{n}, \quad n \geq 1 .
$$

We pass from relation (111) to a recurrence relation of the first order in the space $\mathbb{R}^{2}$, namely

$$
\widetilde{S}_{1}=\Theta_{1}, \quad \widetilde{S}_{n}=B_{n} \widetilde{S}_{n-1}+\Theta_{n}, \quad n \geq 2,
$$

where

$$
\widetilde{S}_{n}=\left(\begin{array}{c}
S_{n} \\
S_{n-1}
\end{array}\right), \quad B_{n}=\left(\begin{array}{cc}
1+\alpha_{n} & -\alpha_{n} \\
1 & 0
\end{array}\right), \quad \Theta_{n}=\left(\begin{array}{c}
\beta_{n} \gamma_{n} \\
0
\end{array}\right), \quad n \geq 1 .
$$

The above notation implies that the random series (2) converges almost surely if and only if the $\operatorname{limit}_{n \rightarrow \infty} \lim _{n}$ exists almost surely.

Now we transform the recurrence relation (12) to the following form:

$$
\widetilde{S}_{n}=\left(\prod_{j=n}^{2} B_{j}\right) \Theta_{1}+\left(\prod_{j=n}^{3} B_{j}\right) \Theta_{2}+\cdots+B_{n} \Theta_{n-1}+\Theta_{n}, \quad n \geq 1,
$$

where $\prod_{j=n}^{k} B_{j}=B_{n} B_{n-1} \ldots B_{k}, k \leq n$.

Using mathematical induction, we prove that

$$
\prod_{j=n}^{k} B_{j}=\left(\begin{array}{cc}
1+\sum_{l=1}^{n-k+1}\left(\prod_{j=k}^{k+l-1} \alpha_{j}\right) & -\sum_{l=1}^{n-k+1}\left(\prod_{j=k}^{k+l-1} \alpha_{j}\right) \\
1+\sum_{l=1}^{n-k}\left(\prod_{j=k}^{k+l-1} \alpha_{j}\right) & -\sum_{l=1}^{n-k}\left(\prod_{j=k}^{k+l-1} \alpha_{j}\right)
\end{array}\right), \quad 2 \leq k \leq n .
$$


Recalling definition (5) we obtain

$$
\prod_{j=n}^{k} B_{j}=\left(\begin{array}{cc}
A^{\prime}(n, k-1) & 1-A^{\prime}(n, k-1) \\
A^{\prime}(n-1, k-1) & 1-A^{\prime}(n-1, k-1)
\end{array}\right), \quad 2 \leq k \leq n .
$$

Turning back to the proof of the necessity we will show that conditions 1)-3) of Theorem 3.1 follow from the almost sure convergence of the random series (2).

Let the random series (2) converge almost surely. Then, as proved in the paper [4, conditions 1) and 2) of Theorem 3.1 hold.

To prove condition 3), we apply the contraction principle in the space of convergent sequences (see [2, 3]). Fix an arbitrary sequence $\left(m_{j}\right)$ of the class $\mathfrak{R}^{\infty}$.

Consider a family of random vectors $\left(Z_{n, k} ; n, k \geq 1\right)$ defined by

$$
Z_{n, k}= \begin{cases}\left(\prod_{j=n}^{k+1} B_{j}\right) \Theta_{k}, & 1 \leq k \leq n-1, \\ \Theta_{k}, & k=n, \\ \overrightarrow{0}, & k>n,\end{cases}
$$

where $\overrightarrow{0}$ is the zero vector in the space $\mathbb{R}^{2}$. This family of random variables satisfies the following two conditions:

a) for all $n \geq 1$, the series $\sum_{k=1}^{\infty} Z_{n, k}$ converges almost surely in the norm of the space $\mathbb{R}^{2}$

b) the sequences $W_{k}=\left(Z_{n, k}, n \geq 1\right), k \geq 1$, are independent and symmetric as random elements of the space of sequences. Moreover,

$$
\widetilde{S}_{n}=\sum_{k=1}^{\infty} Z_{n, k}, \quad n \geq 1 .
$$

Along with the family $\left(Z_{n, k} ; n, k \geq 1\right)$ consider the family of contractions $\left(\beta_{n, k} ; n, k \geq 1\right)$, where

$$
\beta_{n, k}= \begin{cases}1, & n=m_{j+1}, m_{j}<k \leq m_{j+1}, j \geq 1, \\ 0, & \text { otherwise. }\end{cases}
$$

Since the random series (2) converges almost surely, the limit $\lim _{n \rightarrow \infty} \widetilde{S}_{n}$ exists almost surely. This implies that the sequence of vectors $\left(\sum_{k=1}^{\infty} Z_{n, k}\right)$ converges almost surely in the space $\mathbb{R}^{2}$ and, moreover,

$$
\left\|\beta_{n, k} Z_{n, k}\right\| \underset{n \rightarrow \infty}{\longrightarrow} 0, \quad k \geq 1 \quad \text { almost surely. }
$$

According to the contraction principle (see Corollary 2.7.1 in [2]) this implies that

$$
\left\|\sum_{k=1}^{\infty} \beta_{n, k} Z_{n, k}\right\| \underset{n \rightarrow \infty}{\longrightarrow} 0 \quad \text { almost surely. }
$$

If one uses the above notation, the latter relation can be rewritten as follows:

$$
\left\|\sum_{k=m_{j}+1}^{m_{j+1}}\left(\prod_{i=m_{j+1}}^{k+1} B_{i}\right) \Theta_{k}\right\| \underset{j \rightarrow \infty}{\longrightarrow} 0 \text { almost surely, }
$$

which is equivalent to

$$
\left\|\left(\begin{array}{c}
\sum_{k=m_{j}+1}^{m_{j+1}} A\left(m_{j+1}, k\right) \gamma_{k} \\
\sum_{k=m_{j}+1}^{m_{j+1}-1} A\left(m_{j+1}-1, k\right) \gamma_{k}
\end{array}\right)\right\| \underset{j \rightarrow \infty}{\longrightarrow} 0 \text { almost surely }
$$

in view of representation (14). 
Since $\left(\gamma_{k}\right)$ is a standard Gaussian sequence, the sum

$$
\sum_{k=m_{j}+1}^{m_{j+1}} A\left(m_{j+1}, i\right) \gamma_{k}
$$

is a centered Gaussian random variable for all $j \geq 1$. Moreover, the random variables

$$
\sum_{i=m_{j_{1}}+1}^{m_{j_{1}+1}} A\left(m_{j_{1}+1}, i\right) \gamma_{i} \text { and } \sum_{i=m_{j_{2}}+1}^{m_{j_{2}+1}} A\left(m_{j_{2}+1}, i\right) \gamma_{i}
$$

are independent whenever $j_{1} \neq j_{2}$. Thus, in view of (15), Lemma 2.1 implies that

$$
\sum_{j=1}^{\infty} \exp \left\{-\frac{\varepsilon}{\sum_{i=m_{j}+1}^{m_{j+1}} A\left(m_{j+1}, i\right)^{2}}\right\}<\infty
$$

for all $\varepsilon>0$.

Therefore condition 3) of Theorem 3.1 holds, and the necessity of the almost sure convergence of series (2) for the assumptions 1)-3) in Theorem 3.1] is proved.

Now we prove the sufficiency part of the theorem. We show that assumptions 1)-3) imply the almost sure convergence of series (2). We distinguish between the following two cases:

I) all coefficients are nonzero, that is, $\alpha_{n} \neq 0, n \geq 1$;

II) there are some zero coefficients $\alpha_{n}$.

Case I). Let all coefficients be nonzero, that is, $\alpha_{n} \neq 0, n \geq 1$. Then all matrices $B_{n}$, $n \geq 1$, involved in representation (13) are nonsingular, that is, $\operatorname{det} B_{n} \neq 0, n \geq 1$. This means that one can pass from relation (13) to the following one:

$$
\begin{gathered}
\widetilde{S}_{n}=\left(\prod_{j=n}^{2} B_{j}\right)\left(\Theta_{1}+B_{2}^{-1} \Theta_{2}+\left(B_{2}^{-1} B_{3}^{-1}\right) \Theta_{3}+\cdots+\left(B_{2}^{-1} B_{3}^{-1} \cdots B_{n}^{-1}\right) \Theta_{n}\right) \\
n \geq 1
\end{gathered}
$$

where $B_{k}^{-1}$ is the inverse matrix for $B_{k}, k \geq 1$.

Now we represent the sequence $\left(\widetilde{S}_{n}\right)$ as a sequence of sums of independent random vectors with an operator normalization (see [2, 3]), namely

$$
\widetilde{S}_{n}=\mathcal{A}_{n} \sum_{k=1}^{n} X_{k}=\mathcal{A}_{n} \Xi_{n}, \quad n \geq 1,
$$

where

$$
\begin{gathered}
\Xi_{n}=\sum_{k=1}^{n} X_{k}, \quad n \geq 1, \\
X_{1}=\Theta_{1}, \quad X_{k}=\left(\prod_{j=2}^{k} B_{j}^{-1}\right) \Theta_{k}, \quad k \geq 2, \\
\mathcal{A}_{1}=I, \quad \mathcal{A}_{n}=\prod_{j=n}^{2} B_{j}=\left(\begin{array}{cc}
A^{\prime}(n, 1) & 1-A^{\prime}(n, 1) \\
A^{\prime}(n-1,1) & 1-A^{\prime}(n-1,1)
\end{array}\right), \quad n \geq 2,
\end{gathered}
$$

and where $I$ is the unit $2 \times 2$ matrix.

Note that $\left(X_{n}\right)$ is a sequence of independent centered Gaussian random vectors in the space $\mathbb{R}^{2}$. Thus the sequence $\left(\widetilde{S}_{n}\right)$ is represented in the form of the sequence $\left(\mathcal{A}_{n} \Xi_{n}\right)$ of sums of independent random vectors with an operator normalization in the space $\mathbb{R}^{2}$. 
This implies that the random series (2) converges almost surely if $\left(\mathcal{A}_{n} \Xi_{n}\right) \in c\left(\mathbb{R}^{2}\right)$ almost surely. Now we apply Theorem 2.1 to the sequence $\left(\mathcal{A}_{n} \Xi_{n}\right)$.

Let the series (8) converge for all $k \geq 1$. This means that the limit

$$
\lim _{n \rightarrow \infty} A(n, k)=A(\infty, k)
$$

exists for all $k \geq 1$. Since

$$
\mathcal{A}_{n} X_{k}=B_{n} B_{n-1} \cdots B_{k+1} \Theta_{k}=\left(\begin{array}{c}
A(n, k) \gamma_{k} \\
A(n-1, k) \gamma_{k}
\end{array}\right), \quad k, n \geq 1,
$$

we have $\left(\mathcal{A}_{n} X_{k}\right) \in c\left(\mathbb{R}^{2}\right)$ almost surely for all $k \geq 1$, whence condition $\left.\mathrm{A}\right)$ of Theorem 2.1 follows. Moreover,

$$
Y_{k}=\lim _{n \rightarrow \infty} \mathcal{A}_{n} X_{k}=\lim _{n \rightarrow \infty}\left(\begin{array}{c}
A(n, k) \gamma_{k} \\
A(n-1, k) \gamma_{k}
\end{array}\right)=\left(\begin{array}{c}
A(\infty, k) \gamma_{k} \\
A(\infty, k) \gamma_{k}
\end{array}\right), \quad k \geq 1
$$

Let condition (9) hold. Then the random series

$$
\sum_{k=1}^{\infty} A(\infty, k) \gamma_{k}
$$

converges almost surely. Since

$$
\sum_{k=1}^{\infty} Y_{k}=\sum_{k=1}^{\infty}\left(\begin{array}{l}
A(\infty, k) \gamma_{k} \\
A(\infty, k) \gamma_{k}
\end{array}\right)
$$

we conclude that the series $\sum_{k=1}^{\infty} Y_{k}$ converges almost surely in the space $\mathbb{R}^{2}$. Therefore condition B) of Theorem 2.1 also holds.

Finally we show that condition $\mathrm{C}$ ) of Theorem 2.1 holds. Consider an arbitrary sequence $\left(m_{j}\right)$ of the class $\mathfrak{R}^{\infty}$. Then

$$
\begin{aligned}
\mathcal{A}_{m_{j+1}}\left(\Xi_{m_{j+1}}-\Xi_{m_{j}}\right) & =\left(\prod_{j=m_{j+1}}^{m_{j}+2} B_{j}\right) \Theta_{m_{j}+1}+\cdots+B_{m_{j+1}} \Theta_{m_{j+1}-1}+\Theta_{m_{j+1}} \\
& =\left(\begin{array}{c}
\sum_{i=m_{j}+1}^{m_{j+1}} A\left(m_{j+1}, i\right) \gamma_{i} \\
\sum_{i=m_{j}+1}^{m_{j+1}-1} A\left(m_{j+1}-1, i\right) \gamma_{i}
\end{array}\right), \quad j \geq 1 .
\end{aligned}
$$

Assume that condition (10) holds. Then, according to Lemma 2.1,

$$
\lim _{j \rightarrow \infty}\left|\sum_{i=m_{j}+1}^{m_{j+1}} A\left(m_{j+1}, i\right) \gamma_{i}\right|=0 \text { almost surely. }
$$

This together with (16) implies that

$$
\lim _{j \rightarrow \infty}\left\|\mathcal{A}_{m_{j+1}}\left(\Xi_{m_{j+1}}-\Xi_{m_{j}}\right)\right\|=0 \quad \text { almost surely, }
$$

whence condition $\mathrm{C}$ ) of Theorem 2.1 follows.

Hence all the conditions of Theorem 2.1 hold and thus series (2) converges almost surely. Therefore Theorem 3.1 is proved for the case where all coefficients $\alpha_{n}, n \geq 1$, are nonzero.

Case II). Now let there be some zero coefficients among $\alpha_{n}, n \geq 1$.

The case where there is only a finite number of zeros in the sequence $\alpha_{n}, n \geq 1$, reduces to Case I).

Assume that there is an infinite number of zeros in the sequence $\alpha_{n}, n \geq 1$. The method to prove the result in this case is to construct an auxiliary Gaussian Markov 
sequence $\left(\widehat{\xi}_{k}\right)$ with nonzero coefficients $\widehat{\alpha}_{n}, n \geq 1$, such that all the assumptions of Theorem 3.1 hold for it and the two series

$$
\sum_{k=1}^{\infty} \widehat{\xi}_{k} \text { and } \sum_{k=1}^{\infty} \xi_{k}
$$

converge or diverge simultaneously. A similar method, called the disturbed coefficients method, is introduced in [2, 3].

Along with sequence (1), consider a centered Gaussian Markov sequence $\left(\widehat{\xi}_{k}\right)$ given by the following recurrence relation of the first order:

$$
\widehat{\xi}_{0}=0, \quad \widehat{\xi}_{k}=\widehat{\alpha}_{k} \widehat{\xi}_{k-1}+\beta_{k} \gamma_{k}, \quad k \geq 1,
$$

where the sequences $\left(\beta_{k}\right)$ and $\left(\gamma_{k}\right)$ are the same as in relation (1), while

$$
\widehat{\alpha}_{k}= \begin{cases}\alpha_{k} & \text { if } \alpha_{k} \neq 0 \\ \varepsilon_{k} & \text { if } \alpha_{k}=0\end{cases}
$$

The sequence $\left(\varepsilon_{k}\right)$ is chosen such that $\varepsilon_{k}>0, k \geq 1$, and

$$
\left|\prod_{j=k+1}^{k+l} \widehat{\alpha}_{j}-\prod_{j=k+1}^{k+l} \alpha_{j}\right| \leq \frac{2^{-k} \cdot 2^{-l}}{\tau_{k}}, \quad k, l \geq 1,
$$

where

$$
\tau_{k}= \begin{cases}1 & \text { if } \beta_{k} \in[0,1) \\ \beta_{k} & \text { if } \beta_{k} \in[1, \infty)\end{cases}
$$

First we show that such a sequence $\left(\varepsilon_{k}\right)$ exists. We enumerate the indices $k \geq 1$ for which $\alpha_{k}=0$. The resulting sequence is denoted by $\left(n_{i}\right), i \geq 1$, that is, $\alpha_{n_{i}}=0, i \geq 1$.

Now we choose the sequence $\left(\varepsilon_{n_{i}}\right)$ such that

$$
\varepsilon_{n_{i}} \leq \frac{2^{-n_{(i+1)}}}{\delta_{n_{i}} \prod_{j=1}^{n_{i}} \tau_{j}}, \quad i \geq 1
$$

where

$$
\delta_{n_{i}}= \begin{cases}1 & \text { if } \Delta_{n_{i}} \in(0,1) \\ \Delta_{n_{i}} & \text { if } \Delta_{n_{i}} \in[1, \infty)\end{cases}
$$

and

$$
\Delta_{n_{i}}=\sup _{s \geq n_{(i-1)}+1, t \leq n_{(i+1)}-1}\left|\prod_{m=s}^{n_{i}-1} \alpha_{m} \cdot \prod_{m=n_{i}+1}^{t} \alpha_{m}\right|, \quad i \geq 1 .
$$

Fix $k \geq 1$. Note that

$$
\left|\prod_{j=k+1}^{k+l} \widehat{\alpha}_{j}-\prod_{j=k+1}^{k+l} \alpha_{j}\right|=0, \quad l \geq 1,
$$

if $\alpha_{j} \neq 0$ for all $k+1 \leq j \leq k+l$. Thus we may restrict the consideration to the case where there exists at least one number $n_{i} \in\{k+1, k+2, \ldots, k+l\}$ such that $\alpha_{n_{i}}=0$. 
Then

$$
\begin{aligned}
\left|\prod_{j=k+1}^{k+l} \widehat{\alpha}_{j}-\prod_{j=k+1}^{k+l} \alpha_{j}\right| & =\left|\prod_{j=k+1}^{k+l} \widehat{\alpha}_{j}\right|=\prod_{k+1 \leq j \leq k+l: \alpha_{j} \neq 0} \cdot \prod_{i: k+1 \leq n_{i} \leq k+l} \varepsilon_{n_{i}} \\
& \leq \mid \prod_{k+1 \leq j \leq k+l: \alpha_{j} \neq 0} \alpha_{j} \cdot \prod_{i: k+1 \leq n_{i} \leq k+l} \frac{2^{-n_{(i+1)}}}{\delta_{n_{i}} \prod_{j=1}^{n_{i}} \tau_{j}} \\
& \leq \frac{1}{\tau_{k}} \cdot \prod_{i: k+1 \leq n_{i} \leq k+l} 2^{-n_{(i+1)}} \\
& \leq \frac{2^{-(k+l)}}{\tau_{k}}
\end{aligned}
$$

for all $l \geq 1$. This means that inequalities (17) hold if the sequence $\left(\varepsilon_{k}\right)$ is chosen as indicated above.

We continue the proof of Theorem 3.1 for the case II). We show that assumptions $1)-3)$ of this theorem hold for the sequence $\left(\widehat{\xi}_{k}\right)$.

Indeed, inequalities (17) imply that

$$
\begin{aligned}
\left|\prod_{j=k+1}^{k+l} \widehat{\alpha}_{j}\right| & \leq\left|\prod_{j=k+1}^{k+l} \widehat{\alpha}_{j}-\prod_{j=k+1}^{k+l} \alpha_{j}\right|+\left|\prod_{j=k+1}^{k+l} \alpha_{j}\right| \\
& \leq \frac{2^{-k} \cdot 2^{-l}}{\tau_{k}}+\left|\prod_{j=k+1}^{k+l} \alpha_{j}\right|
\end{aligned}
$$

for $k, l \geq 1$. Note that the series

$$
\sum_{l=1}^{\infty} \frac{2^{-k} \cdot 2^{-l}}{\tau_{k}}=\frac{2^{-k}}{\tau_{k}} \sum_{l=1}^{\infty} 2^{-l}
$$

converges for all $k \geq 1$. Moreover,

$$
\sum_{l=1}^{\infty}\left|\prod_{j=k+1}^{k+l} \alpha_{j}\right|<\infty
$$

for all $k \geq 1$, since the latter series has only a finite number of nonzero terms. This implies that

$$
\sum_{l=1}^{\infty}\left|\prod_{j=k+1}^{k+l} \widehat{\alpha}_{j}\right|<\infty
$$

for all $k \geq 1$. Hence condition 1 ) of Theorem 3.1 holds.

Next we show that assumption 2) of Theorem 3.1 holds for the sequence $\left(\widehat{\xi}_{k}\right)$, that is,

$$
\sum_{k=1}^{\infty}(\widehat{A}(\infty, k))^{2}<\infty
$$


where the numbers $\widehat{A}(\infty, k)$ are defined by equality (7) and where the coefficients $\widehat{\alpha}_{k}$ are used instead of the coefficients $\alpha_{k}$. Since

$$
\begin{aligned}
(\widehat{A}(\infty, k))^{2} & =((\widehat{A}(\infty, k)-A(\infty, k))+A(\infty, k))^{2} \\
& \leq 2(\widehat{A}(\infty, k)-A(\infty, k))^{2}+2(A(\infty, k))^{2} \\
& =2\left(\sum_{l=1}^{\infty}\left(\prod_{j=k+1}^{k+l} \widehat{\alpha}_{j}-\prod_{j=k+1}^{k+l} \alpha_{j}\right)\right)^{2} \beta_{k}^{2}+2(A(\infty, k))^{2} \\
& \leq 2\left(\sum_{l=1}^{\infty} \frac{2^{-k} \cdot 2^{-l}}{\tau_{k}}\right)^{2} \beta_{k}^{2}+2(A(\infty, k))^{2} \\
& \leq 2 \cdot 4^{-k}\left(\sum_{l=1}^{\infty} 2^{-l}\right)^{2}+2(A(\infty, k))^{2}=2 \cdot 4^{-k}+2(A(\infty, k))^{2},
\end{aligned}
$$

condition (9) implies relation (18), which means that condition 2) of Theorem 3.1 holds, indeed.

Finally we show that assumption 3 ) of Theorem 3.1 holds for the sequence $\left(\widehat{\xi}_{k}\right)$. Fix an arbitrary sequence $\left(m_{j}\right)$ of the class $\Re^{\infty}$. According to inequalities (17),

$$
\begin{aligned}
& \mathrm{E}\left|\sum_{k=m_{j}+1}^{m_{j+1}} \sum_{l=1}^{m_{j+1}}\left(\prod_{i=k+1}^{k+l} \widehat{\alpha}_{i}-\prod_{i=k+1}^{k+l} \alpha_{i}\right) \beta_{k} \gamma_{k}\right| \\
& \quad \leq \mathrm{E}\left|\gamma_{k}\right| \cdot \sum_{k=m_{j}+1}^{m_{j+1}}\left(\sum_{l=1}^{m_{j+1}} \frac{2^{-k} \cdot 2^{-l}}{\tau_{k}}\right) \beta_{k} \\
& \quad \leq \sqrt{\frac{2}{\pi}} \sum_{k=m_{j}+1}^{m_{j+1}} 2^{-k}\left(1-\left(\frac{1}{2}\right)^{m_{j+1}}\right) \\
& \quad=\sqrt{\frac{2}{\pi}}\left(1-\left(\frac{1}{2}\right)^{m_{j+1}}\right) \cdot\left(1-\left(\frac{1}{2}\right)^{m_{j+1}-m_{j}}\right) \cdot\left(\frac{1}{2}\right)^{m_{j}} \\
& \leq \sqrt{\frac{2}{\pi}}\left(\frac{1}{2}\right)^{m_{j}}
\end{aligned}
$$

for all $j \geq 1$, whence

$$
\sum_{j=1}^{\infty} \mathrm{E}\left|\sum_{k=m_{j}+1}^{m_{j+1}} \sum_{l=1}^{m_{j+1}}\left(\prod_{i=k+1}^{k+l} \widehat{\alpha}_{i}-\prod_{i=k+1}^{k+l} \alpha_{i}\right) \beta_{k} \gamma_{k}\right|<\infty .
$$

This means that the random series

$$
\sum_{j=1}^{\infty}\left|\sum_{k=m_{j}+1}^{m_{j+1}} \sum_{l=1}^{m_{j+1}}\left(\prod_{i=k+1}^{k+l} \widehat{\alpha}_{i}-\prod_{i=k+1}^{k+l} \alpha_{i}\right) \beta_{k} \gamma_{k}\right|
$$

converges almost surely and

$$
\left|\sum_{k=m_{j}+1}^{m_{j+1}} \sum_{l=1}^{m_{j+1}}\left(\prod_{i=k+1}^{k+l} \widehat{\alpha}_{i}-\prod_{i=k+1}^{k+l} \alpha_{i}\right) \beta_{k} \gamma_{k}\right| \underset{j \rightarrow \infty}{\longrightarrow} 0 \text { almost surely. }
$$


The latter relation can be rewritten as follows:

$$
\left|\sum_{k=m_{j}+1}^{m_{j+1}}\left(\widehat{A}\left(m_{j+1}, k\right)-A\left(m_{j+1}, k\right)\right) \gamma_{k}\right| \underset{j \rightarrow \infty}{\longrightarrow} 0 \text { almost surely }
$$

where the numbers $\widehat{A}(n, k), n, k \geq 1$, are defined by equality (6), in which the coefficients $\widehat{\alpha}_{k}$ are used instead of the coefficients $\alpha_{k}$.

Recalling condition (10) we get from Lemma 2.1 that

$$
\left|\sum_{k=m_{j}+1}^{m_{j+1}} A\left(m_{j+1}, k\right) \gamma_{k}\right| \underset{j \rightarrow \infty}{\longrightarrow} 0 \text { almost surely. }
$$

Thus relation (19) yields

$$
\left|\sum_{k=m_{j}+1}^{m_{j+1}} \widehat{A}\left(m_{j+1}, k\right) \gamma_{k}\right| \underset{j \rightarrow \infty}{\longrightarrow} 0 \text { almost surely. }
$$

According to Lemma 2.1.

$$
\sum_{j=1}^{\infty} \exp \left\{-\frac{\varepsilon}{\sum_{k=m_{j}+1}^{m_{j+1}} \widehat{A}\left(m_{j+1}, k\right)^{2}}\right\}<\infty .
$$

Hence assumption 3) of Theorem 3.1 holds. Therefore the sequence $\left(\widehat{\xi}_{k}\right)$ satisfies all the assumptions of Theorem 3.1, whence we conclude that the series $\sum_{k=1}^{\infty} \widehat{\xi}_{k}$ converges almost surely in view of the case I) proved above.

Now we prove that the almost sure convergence of the series $\sum_{k=1}^{\infty} \xi_{k}$ is equivalent to that of the series $\sum_{k=1}^{\infty} \widehat{\xi}_{k}$. Since

$$
\begin{aligned}
\mathrm{E}\left|\widehat{\xi}_{k}-\xi_{k}\right| & =\mathrm{E}\left|\sum_{i=1}^{k-1}\left(\prod_{j=i+1}^{k} \widehat{\alpha}_{j}\right) \beta_{i} \gamma_{i}-\sum_{i=1}^{k-1}\left(\prod_{j=i+1}^{k} \alpha_{j}\right) \beta_{i} \gamma_{i}\right| \\
& =\mathrm{E}\left|\sum_{i=1}^{k-1}\left(\prod_{j=i+1}^{k} \widehat{\alpha}_{j}-\prod_{j=i+1}^{k} \alpha_{j}\right) \beta_{i} \gamma_{i}\right| \\
& \leq \mathrm{E}\left|\gamma_{i}\right| \cdot \sum_{i=1}^{k-1}\left|\prod_{j=i+1}^{k} \widehat{\alpha}_{j}-\prod_{j=i+1}^{k} \alpha_{j}\right| \beta_{i} \\
& \leq \sqrt{\frac{2}{\pi}} \cdot \sum_{i=1}^{k-1} \frac{2^{-i} \cdot 2^{-(k-i)}}{\tau_{i}} \beta_{i} \leq \sqrt{\frac{2}{\pi}} \cdot(k-1) \cdot 2^{-k},
\end{aligned}
$$

the series $\sum_{k=1}^{\infty} \mathrm{E}\left|\widehat{\xi}_{k}-\xi_{k}\right|$ converges. This implies the almost sure convergence of the random series $\sum_{k=1}^{\infty}\left|\widehat{\xi}_{k}-\xi_{k}\right|$, whence we establish the almost sure convergence of the random series $\sum_{k=1}^{\infty}\left(\widehat{\xi}_{k}-\xi_{k}\right)$. Therefore the almost sure convergence of the series $\sum_{k=1}^{\infty} \xi_{k}$ is equivalent to that of the series $\sum_{k=1}^{\infty} \widehat{\xi}_{k}$.

Since the random series $\sum_{k=1}^{\infty} \widehat{\xi}_{k}$ converges almost surely, we conclude that $\sum_{k=1}^{\infty} \xi_{k}$ converges almost surely, too. Thus Theorem 3.1 is valid for the case II), as well, and the proof is complete. 


\section{Corollaries}

Corollary 4.1. Let $\alpha_{k}=0$ for all $k \geq 1$, that is, let $\left(\xi_{k}\right)$ be a sequence of independent Gaussian random variables. The random series (2) converges almost surely if and only if

$$
\sum_{k=1}^{\infty} \beta_{k}^{2}<\infty
$$

Proof. In this case,

and

$$
A(n, k)=\beta_{k}, \quad n \geq 1,
$$

$$
A(\infty, k)=\beta_{k}, \quad k \geq 1 .
$$

Thus assumptions 1) and 2) of Theorem 3.1 are equivalent to condition (20).

We show that condition (20) implies assumption 3) of Theorem 3.1] Consider an arbitrary sequence $\left(m_{j}\right)$ of the class $\mathfrak{R}^{\infty}$. Then

$$
\exp \left\{-\frac{\varepsilon}{\sum_{i=m_{j}+1}^{m_{j+1}} \beta_{i}^{2}}\right\} \leq \frac{1}{\varepsilon}\left(\sum_{i=m_{j}+1}^{m_{j+1}} \beta_{i}^{2}\right)
$$

for all $j \geq 1$. The convergence of the series

$$
\sum_{j=1}^{\infty}\left(\sum_{i=m_{j}+1}^{m_{j+1}} \beta_{i}^{2}\right)
$$

follows directly from condition (20). Thus assumption 3) of Theorem 3.1 holds. According to Theorem 3.1, the random series (2) converges almost surely if and only if condition (20) is satisfied. Corollary 4.1 is proved.

Note that Corollary 4.1 coincides with the classical two series theorem in the case of Gaussian random variables.

Corollary 4.2. Let $\beta_{1}=1$ and $\beta_{k}=0$ for all $k \geq 2$. The random series (2) converges almost surely if and only if the series

converges.

$$
\sum_{l=1}^{\infty} \prod_{j=2}^{l+1} \alpha_{j}
$$

Proof. Corollary 4.2 follows obviously from Theorem 3.1

Corollary 4.3. Let $\alpha_{k} \geq 0$ for all $k \geq 2$. The random series (2) converges almost surely if and only if

1) series (8) converges for all $k \geq 1$,

2) relation (9) holds.

Proof. Assumptions 1) and 2) of Corollary 4.3 coincide with assumptions 1) and 2) of Theorem 3.1. respectively. Hence we need to show that assumption 2) of Theorem 3.1] implies assumption 3) of the same theorem and then Corollary 4.3 follows.

Consider an arbitrary sequence $\left(m_{j}\right)$ of the class $\mathfrak{R}^{\infty}$. Since $\alpha_{k} \geq 0$ for $k \geq 1$,

$$
\left(A\left(m_{j+1}, i\right)\right)^{2} \leq(A(\infty, i))^{2}, \quad i \geq 1
$$

for all $j \geq 1$, whence

$$
\exp \left\{-\frac{\varepsilon}{\sum_{i=m_{j}+1}^{m_{j+1}}\left(A\left(m_{j+1}, i\right)\right)^{2}}\right\} \leq \frac{1}{\varepsilon}\left(\sum_{i=m_{j}+1}^{m_{j+1}}\left(A\left(m_{j+1}, i\right)\right)^{2}\right) \leq \frac{1}{\varepsilon}\left(\sum_{i=m_{j}+1}^{m_{j+1}}(A(\infty, i))^{2}\right)
$$


for all $j \geq 1$. The convergence of the series

$$
\sum_{j=1}^{\infty}\left(\sum_{i=m_{j}+1}^{m_{j+1}}(A(\infty, i))^{2}\right)
$$

follows from relation (9). Therefore assumption 3) of Theorem 3.1 holds. Applying Theorem 3.1 we complete the proof of Corollary 4.3 .

Remark 4.1. Note that Corollary 4.3 coincides with a result of the paper [4].

Consider a few sufficient conditions following from Theorem 3.1

Corollary 4.4. Let the following three conditions hold:

1) series (8) converges for all $k \geq 1$,

2) relation (9) is satisfied,

3) for all $k \geq 1$, there exists a positive constant $M$ such that

$$
\sup _{n \geq 1}|A(n, k)|<M \cdot|A(\infty, k)|, \quad k \geq 1
$$

Then the random series (2) converges almost surely.

Proof. Assumptions 1) and 2) of Corollary 4.4 coincide with assumptions 1) and 2) of Theorem 3.1, respectively. Hence we need to prove assumption 3) of Theorem 3.1 and then Corollary 4.4 follows.

Consider an arbitrary sequence $\left(m_{j}\right)$ of the class $\mathfrak{R}^{\infty}$. Then

$$
\begin{aligned}
\exp \left\{-\frac{\varepsilon}{\sum_{i=m_{j}+1}^{m_{j+1}}\left(A\left(m_{j+1}, i\right)\right)^{2}}\right\} & \leq \frac{1}{\varepsilon}\left(\sum_{i=m_{j}+1}^{m_{j+1}}\left(A\left(m_{j+1}, i\right)\right)^{2}\right) \\
& \leq \frac{M}{\varepsilon}\left(\sum_{i=m_{j}+1}^{m_{j+1}}(A(\infty, i))^{2}\right)
\end{aligned}
$$

for all $j \geq 1$. Reasoning as in the proof of Corollary 4.3, we complete the proof of Corollary 4.4 .

Corollary 4.5. Let the following three conditions hold:

1) series (8) converges for all $k \geq 1$,

2) $\sup _{n, k \geq 1}\left|A^{\prime}(n, k)\right|<\infty$,

3) relation (20) holds.

Then the random series (2) converges almost surely.

Proof. We check the assumptions of Theorem 3.1. Assumptions 1) and 2) of Theorem 3.1 follow directly from assumptions 1) and 2) of Corollary 4.5. We show that assumption 3) of Theorem 3.1 also holds; namely, we prove that relation (10) holds for all $\varepsilon>0$ and all 
sequences $\left(m_{j}\right)$ of the class $\mathfrak{R}^{\infty}$. Since

$$
\begin{aligned}
\exp \left\{-\frac{\varepsilon}{\sum_{i=m_{j}+1}^{m_{j+1}} A\left(m_{j+1}, i\right)^{2}}\right\} & =\exp \left\{-\frac{\varepsilon}{\sum_{i=m_{j}+1}^{m_{j+1}} \beta_{i}^{2} A^{\prime}\left(m_{j+1}, i\right)^{2}}\right\} \\
& \leq \frac{1}{\varepsilon}\left(\sum_{i=m_{j}+1}^{m_{j+1}} \beta_{i}^{2} A^{\prime}\left(m_{j+1}, i\right)^{2}\right) \\
& \leq \frac{1}{\varepsilon}\left(\sum_{i=m_{j}+1}^{m_{j+1}} \beta_{i}^{2}\left(\sup _{n, k \geq 1}\left|A^{\prime}(n, k)\right|\right)^{2}\right) \\
& =\frac{1}{\varepsilon}\left(\sup _{n, k \geq 1}\left|A^{\prime}(n, k)\right|\right)^{2}\left(\sum_{i=m_{j}+1}^{m_{j+1}} \beta_{i}^{2}\right)
\end{aligned}
$$

for all $j \geq 1$, relation (10) holds if the series

$$
\sum_{j=1}^{\infty}\left(\sum_{i=m_{j}+1}^{m_{j+1}} \beta_{i}^{2}\right)
$$

converges. The convergence of the latter series follows from relation (20). Thus assumption 3) of Theorem 3.1 holds and this yields that the random series (2) converges almost surely. This completes the proof of Corollary 4.5 .

Below are some examples of sequences $\left(\alpha_{k}\right)$ satisfying assumptions 1) and 2) of Corollary 4.5 .

Example 4.1. Let $q \in(-1,1)$ be a fixed number and let

$$
\alpha_{k}=q, \quad k \geq 1 \text {. }
$$

Then

$$
A^{\prime}(n, k)=\sum_{l=0}^{n} q^{l}=\frac{1-q^{n-k+1}}{1-q}, \quad 1 \leq k \leq n .
$$

Hence

$$
\sup _{n, k \geq 1}\left|A^{\prime}(n, k)\right|=\frac{2}{1-q}<\infty .
$$

Moreover, series (8) converges for all $k \geq 1$, whence

$$
A^{\prime}(\infty, k)=\sum_{l=0}^{\infty} q^{l}=\frac{1}{1-q}=\text { const }, \quad k \geq 1 .
$$

Thus the random series (2) converges almost surely if relation (20) holds.

Example 4.2. Consider the sequence

$$
\alpha_{k}=-\frac{k-1}{k}, \quad k \geq 1
$$

In this case

$$
A^{\prime}(n, k)=\sum_{l=0}^{n-k}(-1)^{l} \frac{k}{k+l}, \quad 1 \leq k \leq n,
$$

and series (8) is of the form

$$
\sum_{l=0}^{\infty}(-1)^{l} \frac{k}{k+l}, \quad k \geq 1
$$


This series converges conditionally for all $k \geq 1$. Indeed, this series is sign alternating, and the sequence $\left(\frac{k}{k+n}, n \geq 1\right)$ decreases. Moreover,

$$
\lim _{n \rightarrow \infty} \frac{k}{k+n}=0, \quad k \geq 1 .
$$

Further, by the Leibniz test,

$$
0<\frac{1}{k+1} \leq A^{\prime}(2 n, k) \leq A^{\prime}(\infty, k) \leq A^{\prime}(2 n+1, k) \leq 1,
$$

for all $k \geq 1$ and for all $n \geq 1$. Hence

$$
\sup _{n, k \geq 1}\left|A^{\prime}(n, k)\right|=1<\infty .
$$

Therefore the random series (2) converges almost surely if relation (20) holds.

Example 4.3. Let $0<q<1$ be fixed and let

$$
\alpha_{k}=1-\frac{1}{k^{q}}, \quad k \geq 1 .
$$

Note that $\alpha_{k} \geq 0$ for all $k \geq 1$. Thus

$$
1<A^{\prime}(n, k) \leq A^{\prime}(\infty, k), \quad k \geq 1 \text {. }
$$

We show that series (8) converges for all $k \geq 1$ if $0<q<1$. Indeed, series (8) can be rewritten as follows:

$$
\sum_{l=1}^{\infty} \prod_{j=k+1}^{k+l}\left(1-\frac{1}{j^{q}}\right)
$$

Since $0<q<1$, the Raabe test [5] implies that

$$
\lim _{n \rightarrow \infty} n\left(\frac{1}{1-\frac{1}{(n+1)^{q}}}-1\right)=\lim _{n \rightarrow \infty}\left(\frac{n}{(n+1)^{q}-1}\right)=\infty .
$$

Thus series (8) converges for all $k \geq 1$. In addition,

$$
\sup _{n, k \geq 1}\left|A^{\prime}(n, k)\right| \leq \sup _{k \geq 1} A^{\prime}(\infty, k)<A^{\prime}(\infty, 1)<\infty .
$$

Thus the random series (2) converges almost surely if relation (20) holds.

\section{BIBLIOGRAPHY}

1. V. V. Buldygin, The strong law of large numbers and the convergence to zero of Gaussian sequences, Teor. Verojatnost. i Mat. Statist. 19 (1978), 33-41; English transl. in Theor. Probability Math. Statist. 19 (1980), 35-43. MR0494429 (58:13294)

2. V. V. Buldygin and S. A. Solntsev, Functional methods in problems of the summation of random variables, Naukova Dumka, Kiev, 1989. (Russian) MR1007589 (91i:60013)

3. V. V. Buldygin and S. A. Solntsev, Asymptotic Behavior of Linearly Transformed Sums of Random Variables, Kluwer Academic Publishers, Dordrecht, 1997. MR1471203 (98m:60002)

4. V. V. Buldygin and M. K. Runovska, On the convergence of series of autoregressive sequences, Theory Stoch. Process. 15(31) (2009), no. 1, 7-14. MR2603166 (2011a:60116)

5. N. N. Vorob'ev, Theory of Series, Nauka, Moscow, 1979. (Russian) MR.548875 (80i:40001)

Department of Mathematical Analysis and Probability Theory, National Technical University of Ukraine ("KPI"), Peremogy Avenue 37, Kyiv 03056, Ukraine

E-mail address: matan@ntu-kpi.kiev.ua 